IdeAs

Idées d'Amériques

$10 \mid 2017$

États-Unis / Cuba : une nouvelle donne?

\title{
Le Brésil aujourd'hui, la descente aux enfers
}

\section{Christian Azaïs}

\section{(2) OpenEdition}

Journals

Édition électronique

URL : https://journals.openedition.org/ideas/2219

DOI : 10.4000/ideas. 2219

ISSN : 1950-5701

\section{Éditeur}

Institut des Amériques

\section{Référence électronique}

Christian Azaïs, «Le Brésil aujourd'hui, la descente aux enfers », IdeAs [En ligne], 10 | 2017, mis en ligne le 19 décembre 2017, consulté le 19 octobre 2022. URL : http://journals.openedition.org/ideas/2219 ; DOI : https://doi.org/10.4000/ideas.2219

Ce document a été généré automatiquement le 19 octobre 2022

\section{(c) (i) (2)}

Creative Commons - Attribution - Pas d'Utilisation Commerciale - Pas de Modification 4.0 International - CC BY-NC-ND 4.0

https://creativecommons.org/licenses/by-nc-nd/4.0/ 


\title{
Le Brésil aujourd'hui, la descente aux enfers
}

\author{
Christian Azaïs
}

1 Pour illustrer cette rubrique Eclairages, il m'a semblé que seules des Brésilien-ne-s, chercheur-e-s en l'occurrence, avec une longue tradition de réflexion sur la société dans laquelle ils/elles vivent pourraient apporter un regard éclairé sur une situation qui n'arrête pas de se dégrader en termes démocratiques.

2 Ces chercheurs viennent d'horizons disciplinaires différents : du droit du travail, de l'économie et de la sociologie. Je désire les remercier pour leurs différents apports et leurs points de vue multiples sur une situation qui n'est pas neutre d'un point de vue politique. Mon choix s'est porté sur elles et eux car elles/ils illustrent le moment politique dans lequel se situe le Brésil aujourd'hui, après la destitution de la présidente Dilma Rousseff, élue démocratiquement. La violence avec laquelle les droits sociaux minima obtenus grâce à la Constitution progressiste de 1988 sont bafoués est la thématique centrale des contributions présentées ici. En contrepoint, un entretien avec un économiste, défenseur de la réforme de la sécurité sociale, pour qui cette réforme est indispensable sur le plan fiscal.

Il n'en reste pas moins que l'adoption de mesures ultralibérales éculées, la tentative de démantèlement dans un temps extrêmement court de toute protection sociale, menée sous l'impulsion du puissant groupe parlementaire ruralista (représentants des propriétaires terriens), constituent une régression sociale difficilement imaginable dans les pays du Nord en un si court laps de temps. Ces députés et sénateurs - sur un total de 513 parlementaires, 273 se revendiquent de cette mouvance (16 sénateurs et 257 députés), 190 représentent les entrepreneurs et les 50 restant les autres groupes d'intérêt (Jornal Valor Econômico, 15 janvier 2015) - soutiennent nombre de propositions au Congrès national, contraires aux droits indigènes garantis dans la Constitution de 1988, qui depuis près de trente ans protègent la diversité culturelle et environnementale brésilienne. Que désirent-ils? S'approprier les terres indigènes, les unités de conservation, expulser les Indiens restants et pratiquer la culture extensive 
du soja ou d'autres plantes ou d'élevages qui leur garantiront un retour sur investissement important et à court terme.

4 Ceci n'est qu'un exemple des mesures prises par le gouvernement et les parlementaires brésiliens, en défense d'intérêts corporatistes d'où est exclue toute considération qui pourrait servir aux intérêts de la majeure partie de la population.

Pour laisser au lecteur le soin et le plaisir de découvrir par lui-même la teneur des propos présentés dans les quatre articles qui composent cette section Eclairages, je me limiterai à l'indication des thématiques abordées, que je complèterai par un entretien mené au téléphone avec Fabio Giambiagi, économiste - que je remercie pour sa disponibilité - qui défend les mesures adoptées en matière de sécurité sociale. Fort de cet ensemble de considérations, je terminerai par quelques commentaires sur la nature du politique aujourd'hui.

6 Le texte de Ruy Braga met en avant les espoirs déçus d'une globalisation qui, à la fin des années 2000, plaçaient le Sud et les pays émergents - notamment les BRICS (Brésil, Russie, Inde, Chine et Afrique du Sud) - dans le rôle de moteurs de la croissance mondiale. Désormais, l'enjeu est celui de la fin de la société salariale au Brésil. Les attaques contre la société salariale sont la manifestation d'une remise en cause des bases institutionnelles de la légitimité de l'Etat.

7 Liana Carleial centre son argumentaire sur la question du sous-développement et du choix délibéré du gouvernement actuel de contrecarrer toute possibilité d'en sortir à moyen terme. Selon elle, seule la reprise du processus démocratique pourrait constituer un espoir pour les générations futures.

8 Sayonara G.L.C. da Silva analyse les changements dans la législation du travail et pointe les attaques récentes contre les droits des travailleurs et la mise au pas des juges du travail et de la justice du travail. Elle décortique les dispositifs qui remettent en cause la manière de concevoir les relations professionnelles et insiste sur la précarisation croissante du monde du travail.

9 Lena Lavinas s'insurge contre l'idée selon laquelle «la Constitution ne peut rentrer dans le budget de la République ", position qu'elle réfute catégoriquement, car c'est pour elle le moyen de justifier que les droits sociaux contenus dans la Constitution de 1988 appartiennent à un temps révolu et qu'il est grand temps de les revoir... à la baisse, car trop onéreux.

10 En contrepoint à ces contributions, les propos du Professeur Fabio Giambagi ${ }^{1}$, économiste, défenseur tenace de la réforme fiscale voulue par le gouvernement brésilien, pour qui la proposition de réforme fiscale présentée en décembre 2016 était ambitieuse. Elle s'attaquait de front aux pressions fiscales, mais à son grand dam, elle n'a pas passé la barrière du Parlement. En effet, lorsqu'elle est repassée devant le Parlement, au mois d'avril 2017, la proposition originale avait perdu $25 \%$ de son impact sur le plan fiscal. La réforme proposée représentait alors un véritable séisme politique à un moment où le président de la République en exercice, Michel Temer, risquait de perdre son mandat et d'être victime à son tour d'un impeachment, comme sa prédécesseure Dilma Rousseff. Il fallait donc au gouvernement réunir le plus de députés possible susceptibles d'approuver la réforme et éviter à tout prix que les groupes 
populaires ne soient trop pénalisés. Pour ce faire, trois mesures emblématiques ont été écartées :

- le recul de la retraite au-delà de 65 ans, pour certaines catégories de professions et éviter des départs très jeunes

- le passage de la durée de cotisation limitée à 25 ans et non à 15 ans, comme c'est le cas aujourd'hui et

- l'élévation de l'âge de départ à la retraite des travailleuses rurales de 55 à 57 ans.

11 L'abandon de ces trois mesures a redonné une marge de manœuvre au gouvernement, lui permettant de gagner en efficacité sur le plan fiscal tout en préservant ses chances de vote au Congrès. A l'heure qu'il est, alors que le passage à nouveau devant le pouvoir législatif est programmé aux alentours du 20 décembre 2017, la nouvelle proposition qui a été écornée et ne représente plus que $60 \%$ de la proposition initiale - a de fortes chances d'être approuvée avant la fin de l'année 2017. Si elle ne l'était pas, l'année 2018 étant une année électorale, le Brésil risque fort de voir cette réforme tomber aux oubliettes, ce qui aux yeux du professeur Giambiagi serait fort dommageable.

Tous ces textes, en pointant les soubresauts de la démocratie au Brésil aujourd'hui, posent la question de l'avenir du politique dans des « démocraties » où le parlement est soit un parlement croupion, une chambre d'enregistrement des décisions prises par l'exécutif, soit soumis aux caprices d'un individu inondant la sphère médiatique de ses tweets et faisant plusieurs fois par jour des déclarations plus contradictoires les unes que les autres, ce qui contribue à discréditer la démocratie telle qu'elle est entendue et vécue dans les sociétés occidentales contemporaines. Le Brésil n'est en aucun cas une exception dans ce panorama peu propice à l'expression démocratique.

\section{NOTES}

1. Je tiens à remercier M. Fabio Giambiagi pour l'entretien téléphonique qu'il m'a accordé et qui m'a servi à élaborer ces quelques lignes à propos de la réforme de la sécurité sociale, thème dont il est spécialiste.

\section{AUTEUR}

\section{CHRISTIAN AZAÏS}

Christian Azaïs est professeur de sociologie au Conservatoire national des Arts et métiers (Paris) et chercheur au Laboratoire interdisciplinaire pour la sociologie économique (LISE) - UMR 3320, CNAM/Paris. Il a été professeur de science politique à l'Université fédérale de la Paraíba (Brésil), de 1982 à 1994, il mène depuis ce temps des recherches sur le travail, en Amérique latine et en 
France, principalement. Il a co-organisé avec Liana Carleial l'ouvrage La « zone grise » du travail - Dynamiques d'emploi et négociation au Sud et au Nord, paru en novembre 2017 chez Peter Lang. 